\title{
The Prognostic Role of Cancer Stem Cell Markers for Long-term Outcome After Resection of Colonic Liver Metastases
}

\author{
LIDEWIJ SPELT $^{1}$, AGATA SASOR ${ }^{2}$, DANIEL ANSARI ${ }^{1}$, \\ KATARZYNA SAID HILMERSSON ${ }^{2}$ and ROLAND ANDERSSON ${ }^{1}$ \\ ${ }^{1}$ Department of Surgery, Clinical Sciences Lund, Lund University and Skåne University Hospital, Lund, Sweden; \\ ${ }^{2}$ Department of Pathology, Skåne University Hospital, Lund, Sweden
}

\begin{abstract}
Background/Aim: To assess the expression of cancer stem cell (CSC) markers CD44, CD133 and CD24 in colon cancer liver metastases and analyse their predictive value for overall survival (OS) and disease-free survival (DFS) after liver resection. Materials and Methods: Patients operated on for colon cancer liver metastases were included. CSC marker expression was determined through immunohistochemistry analysis. OS and DFS were compared between marker-positive and marker-negative patients. Multivariate analysis was performed to select predictive variables for OS and DFS. Results: CD133positive patients had a worse DFS than CD133-negative patients, with a median DFS of 12 and 25 months $(p=0.051)$. Multivariate analysis selected CD133 expression as a significant predictor for DFS. CD44 and CD24 were not found to predict OS or DFS. Conclusion: CD133 expression in colonic liver metastases is a negative prognostic factor for DFS after liver resection. In the future, CD133 could be used as a biomarker for risk stratification, and possibly for developing novel targeted therapy.
\end{abstract}

Colon cancer has a yearly incidence of 43 per 100,000 inhabitants in Sweden (1). Within 3 years after the diagnosis of colorectal cancer, $29 \%$ of patients will have developed liver metastases (2). In case these can be resected, 5-year survival of up to $60 \%$ is achievable (3).

Prognostic factors have been identified over time and several predictive models have been created for the

This article is freely accessible online.

Correspondence to: Dr. Lidewij Spelt, Department of Surgery, Clinical Sciences Lund, Lund University, Skåne University Hospital, SE-22185 Lund, Sweden. Tel: +46 46173080, e-mail: lidewij.spelt@med.lu.se

Key Words: Colonic neoplasms, disease-free survival, liver resection, metastasis, neoplastic stem cells, survival analysis. stratification of patients into risk categories in order to select management strategies and predict prognosis (4).

Cancer stem cells (CSCs) represent a subset of cancer cells that are presumed to have the properties of normal adult stem cells. These characteristics include the capacity of selfrenewal and asymmetric division, multipotency for differentiation, as well as relatively long cycling times and long-term survival. Research has shown CSCs to be a source of tumour recurrence and resistance to chemotherapy (5). The most often used technique for the identification of CSCs is through the selection of a subpopulation of tumour cells based on the expression of certain cell surface markers (6). Examples of such markers in colorectal cancer are cluster of differentiation (CD) markers CD44, CD133 and CD24 (7-9). CD44 is a cell surface glycoprotein, known to be involved in cell growth, differentiation, adhesion and survival (10). CD133 is a transmembrane glycoprotein, localised mainly at the level of membrane protrusions (11). CD24 is a heavily glycosylated cell surface protein, thought to have various functions in proliferation and cell adhesion (12). In colorectal cancer patients, CD44 and CD24 have been associated with decreased OS $(13,14)$, whilst a metaanalysis showed that CD133 is correlated with a worsened OS and DFS (15). Studies regarding the expression of these markers in colonic liver metastases and its influence on survival after hepatectomy are sparse (16-18). The aim of this study was to assess the expression of CD44, CD133 and $\mathrm{CD} 24$ in colon cancer liver metastases and to analyse their predictive value for OS and DFS after liver resection.

\section{Materials and Methods}

Patients. All consecutive cases of liver resection for colon cancer metastases at Skåne University Hospital, in both Lund and Malmö, Sweden, between January 2006 and December 2014, were identified. From these, patients who also were operated for their primary colon cancer at these hospitals were included in this retrospective cohort study. No patients with rectal cancer were included. Only patients with adenocarcinoma were included, whilst patients with other tumour types, such as mucinous cancers, were excluded. 
Baseline characteristics were collected, including American Society of Anesthesiologists score (ASA-score), pre-operative carcino-embryonic antigen (CEA) level, and grade of complication severity as defined by the Clavien-Dindo classification system (19). Synchronous metastatic disease was defined as metastases occurring within six months after diagnosis of the primary colorectal cancer Preoperative chemotherapy was defined as chemotherapy of which the last cycle was given within three months prior to liver resection. Resections were judged to be minor when a maximum of three separate liver segments as described by Couinaud et al. (20) were resected, or major, if a resection of at least four separate segments was performed (21).

Pathological analysis. Specimens from colon cancer operations were analysed for tumour grade (22), T-stage and N-stage according to the TNM-staging system (23), vascular invasion, perineural invasion and the presence of tumour deposits. Patients were assigned to one of three lymph node ratio (LNR) categories according to our previous study (24). Specimens from the liver resections were analysed for radicality of resection.

Immunohistochemistry (IHC) analysis for the expression of CSC markers was performed on liver specimens of all patients and on colon specimens of a subgroup of 30 randomly selected patients from within the study group.

Formalin-fixed paraffin-embedded specimens of liver metastases and primary colon cancer were sectioned into $4.5 \mu \mathrm{m}$-thick slices for analysis of the expression of CD44, CD133 and CD24 through IHC. IHC procedures were automated using an IHC robot (Autostainer Plus, Dako, Glostrup, Denmark). After heating the sections at $60^{\circ} \mathrm{C}$ for $30 \mathrm{~min}$, they were deparaffinised through xylene and rehydrated in a descending graded series of ethanol solutions. After this the sections were pretreated in a pretreatment module (PT-link, Dako, Glostrup, Denmark) in citrate buffer ( $\mathrm{pH}$ 6.0 , for CD44 and CD133) or Tris/EDTA buffer (pH 8.0, for CD24) at $97^{\circ} \mathrm{C}$ for $20 \mathrm{~min}$ for antigen retrieval. The sections were then inserted into the Autostainer and treated with blocking peroxidase solutions (Envision block, Dako, Glostrup, Denmark), followed by incubation with primary antibodies. Primary monoclonal mouse anti-CD44 antibody (Dako, Glostrup, Denmark) was diluted 1:100, primary mouse anti-CD133 antibody (Miltenyi Biotec, Lund, Sweden) was diluted 1:100 and primary polyclonal goat anti-CD24 (Santa Cruz Biotechnology, Heidelberg, Germany) was diluted 1:50. After a washing step, sections were incubated with secondary antibodies conjugated to HR-peroxidase (anti-rabbit/mouse HRP for CD44 and CD133, anti-goat HRP for CD24, Dako, Glostrup, Denmark). After this, a brown-coloured immunoreaction product was created, using the peroxidase substrate diaminobenzidine (DAB, Dako, Glostrup, Denmark). Hematoxylin was used as background staining, after which sections were dehydrated with alcohol solutions and xylene.

Expression of CD44, CD133 and CD24 was evaluated by an experienced pathologist, who was blinded for other pathological information and clinical outcome. For each individual marker, membranous staining was assessed and considered positive if there was staining of at least $5 \%$ of cells within the microscopic field of vision at 400x magnification, in accordance to previously described cut-off values for the expression of CD44 and CD133 in colorectal cancer (25).

Outcome measures. Within the subgroup of patients in whom IHC analysis was performed on both the primary colon cancer specimens
Table I. Baseline and treatment characteristics in 67 patients operated for colon cancer liver metastases. Median values (range) and absolute frequencies (percentages) are given.

\begin{tabular}{|c|c|c|}
\hline & Value & Missing data \\
\hline Median age in years & $67(39-86)$ & $0 \%$ \\
\hline Male gender & $36(53.7 \%)$ & $0 \%$ \\
\hline \multicolumn{3}{|l|}{ ASA-score } \\
\hline I & $4(6.0 \%)$ & $0 \%$ \\
\hline II & $41(61.2 \%)$ & \\
\hline III & $22(32.8)$ & \\
\hline High tumour grade primary & $10(15.2 \%)$ & $1.5 \%$ \\
\hline Vascular invasion primary & $30(46.9 \%)$ & $4.5 \%$ \\
\hline Perineural invasion primary & $17(27.4 \%)$ & $7.5 \%$ \\
\hline Tumour deposits primary & $14(23.3 \%)$ & $10.4 \%$ \\
\hline \multicolumn{3}{|l|}{ T-stage primary } \\
\hline 1 & $1(1.5 \%)$ & $0 \%$ \\
\hline 2 & $5(7.5 \%)$ & \\
\hline 3 & $45(67.2 \%)$ & \\
\hline 4 & $16(23.9 \%)$ & \\
\hline $\mathrm{N}$-stage primary & 0 & $19(28.4 \%)$ \\
\hline \multicolumn{3}{|l|}{$0 \%$} \\
\hline 1 & $24(35.8 \%)$ & \\
\hline 2 & $24(35.8 \%)$ & \\
\hline \multicolumn{3}{|l|}{ LNR } \\
\hline 0 & $21(31.3 \%)$ & $0 \%$ \\
\hline $0.01-0.18$ & $26(38.8 \%)$ & \\
\hline $0.19-1.00$ & $20(29.9 \%)$ & \\
\hline Median preoperative CEA-level $(\mu \mathrm{g} / \mathrm{L})$ & $5(1-2137)$ & $1.5 \%$ \\
\hline Synchronous liver metastases & $41(61.2 \%)$ & $0 \%$ \\
\hline Median number of liver metastases & $2(1-13)$ & $0 \%$ \\
\hline Median size of largest metastasis $(\mathrm{cm})$ & $2.2(0.5-20)$ & $0 \%$ \\
\hline Bilobar spread & $23(34.3 \%)$ & $0 \%$ \\
\hline Preoperative chemotherapy & $25(37.3 \%)$ & $0 \%$ \\
\hline Liver first operated & $7(10.4 \%)$ & $0 \%$ \\
\hline Extent of liver resection: major & $28(41.8 \%)$ & $0 \%$ \\
\hline Radicality of liver resection & $60(89.6 \%)$ & $0 \%$ \\
\hline Severe complications after liver resection ${ }^{\mathrm{a}}$ & $6(9.0 \%)$ & $0 \%$ \\
\hline Liver metastases CD44 positive & $61(91.0 \%)$ & $0 \%$ \\
\hline Liver metastases CD133 positive & $43(64.2 \%)$ & $0 \%$ \\
\hline Liver metastases CD24 positive & $34(50.7 \%)$ & $0 \%$ \\
\hline
\end{tabular}

aSevere complications as defined by Clavien-Dindo classification grade III-IV. ASA-score: American Society of Anesthesiologists score; LNR: lymph node ratio; CEA: carcinoembryonic antigen.

and the liver metastasis specimens, concordance between expression in the primary cancer and in the liver metastases was determined for each marker.

Within the entire study group, OS and DFS were determined and compared between patients with and without metastatic CSC marker expression. Factors influencing OS and DFS were identified through multivariate analysis.

Data collection and statistical evaluation. Baseline patient characteristics were collected. Number of liver metastases and diameter of the largest metastasis were based on the most recent radiological examination before liver resection, or before start of neo-adjuvant chemotherapy when relevant. 
Spelt et al: Cancer Stem Cell Markers in Colonic Liver Metastases

Table II. Marker expression patterns in primary colon cancer (prim) and liver metastases (met) in 29 patients.

\begin{tabular}{lcccccc}
\hline Marker & prim+/met+ & prim-/met- & prim+/met- & prim-/met+ & Discordance & $p$-Value \\
\hline CD44 & 25 & 0 & 2 & 2 & $4(13.8 \%)$ & 1.00 \\
CD133 & 15 & 5 & 5 & 4 & $9(31.0 \%)$ & 1.00 \\
CD24 & 9 & 6 & 12 & 2 & $14(48.3 \%)$ & 0.013 \\
\hline
\end{tabular}

Statistical analyses were performed using SPSS software (IBM SPSS Statistics for Windows, Version 23.0, IBM Corp., Armonk, NY, USA). Median values (range) describe continuous variables, whereas frequencies (percentages) were calculated for categorical variables. McNemar's test was used to evaluate discordance between marker expression in the primary colon cancers and the liver metastases. Kaplan-Meier analysis was performed to estimate OS and DFS, using the log-rank test to compare groups. $p$-Values $<0.05$ were considered to represent statistical significance.

Cox proportional hazards regression was used to adjust for possible confounding variables by developing a multivariable survival model for both OS and DFS. Inclusion criteria for the full model was a $p$-value $<0.2$ on univariate analysis. The limit for stepwise backward elimination was a $p$-value $<0.1$. A probability level of a random difference of $p$-value $<0.05$ was considered significant. Hazard ratios (HR) and 95\% confidence intervals (95\% CI) were determined

The study was approved by the regional human ethical committee at Lund University, Lund, as well as by the regional biobank centre of southern Sweden, Lund.

This study and its results were reported in accordance with the reporting recommendations for tumour marker prognostic studies (REMARK) as described by McShane et al. (26).

\section{Results}

Patients. A total of 119 patients were considered for this study. A total of 100 patients were considered for further analysis, after the exclusion of two patients where not all liver metastases were treated, one patient who was lost to follow-up, one patient with both a rectal and a colonic primary cancer, eleven patients with mucinous colon carcinoma and four patients with not enough vital tissue for IHC analysis in the liver metastases. Of these, extra liver metastasis tissue for new IHC analyses was available in 67 patients, who were thus included in this study. Patient- and disease characteristics are presented in Table I.

In some cases, data on number of metastases and diameter of the largest metastasis were based on intraoperative findings (4 patients) or pathology reports ( 2 patients), in case new metastases were discovered.

Treatment. All patients underwent liver resection with curative intent for colon cancer metastases. Details on treatment are given in Table I. Preoperative chemotherapy prior to liver resection was administered to 25 out of 67 patients. The median number of chemotherapy cycles was 4 (1-32). Regimens were oxaliplatin-based in 16 patients, irinotecan-based in 7 patients, and including both agents successively in 2 patients. A total of 9 patients also received targeted therapy, such as bevacizumab or cetuximab.

Stem cell marker expression. Out of 67 patients, 61 had positive CD44 expression in their liver metastasis. CD133 and CD24 expression were seen in the liver metastases of 43 and 34 patients, respectively. For each marker, examples of the microscopic appearance of positive liver metastasis tissue are shown in Figure 1.

In a subgroup of 29 patients, since in one patient no sufficient material was available, even the primary colon cancer tissue was analysed through IHC for the expression of CD44, CD133 and CD24. For each marker, examples of the microscopic appearance of positive colon cancer tissue are shown in Figure 1. The patterns of marker expression for matched pairs of colon cancer and liver metastasis samples are shown in Table II. For CD44, CD133 and CD24, there was a discordance between the expression in the primary and in the metastatic cancer tissue in 4, 9 and 14 patients, respectively. This skewness was statistically significant for CD24 only.

Outcome. For long-term outcome, one patient who deceased three days postoperatively was excluded. The remaining 66 patients had a median OS of 63 months with a median follow-up time of 44 months amongst survivors. The median DFS was 16 months and within the follow-up period, 59\% of patients $(n=39)$ developed recurrence. In $59 \%$ of these cases ( $n=23$, equal to $35 \%$ of the total study population) this occurred within the first year after liver resection. The first site of recurrence was intrahepatic only in 18 patients $(46 \%)$, both intrahepatic and extrahepatic in three patients $(8 \%)$, and extrahepatic only in 18 patients $(46 \%)$. The main first extrahepatic site of recurrence was the lungs.

OS and DFS were compared between patients with markernegative and those with marker-positive liver metastases for each CSC marker. OS and DFS curves are shown in Figure 2. The largest differences in both OS and DFS were found when comparing CD133-negative and CD133-positive patients, with a median OS of 79 and 52 months and a median DFS of 25 and 12 months, respectively. For OS, this difference was not 
Primary colon cancer
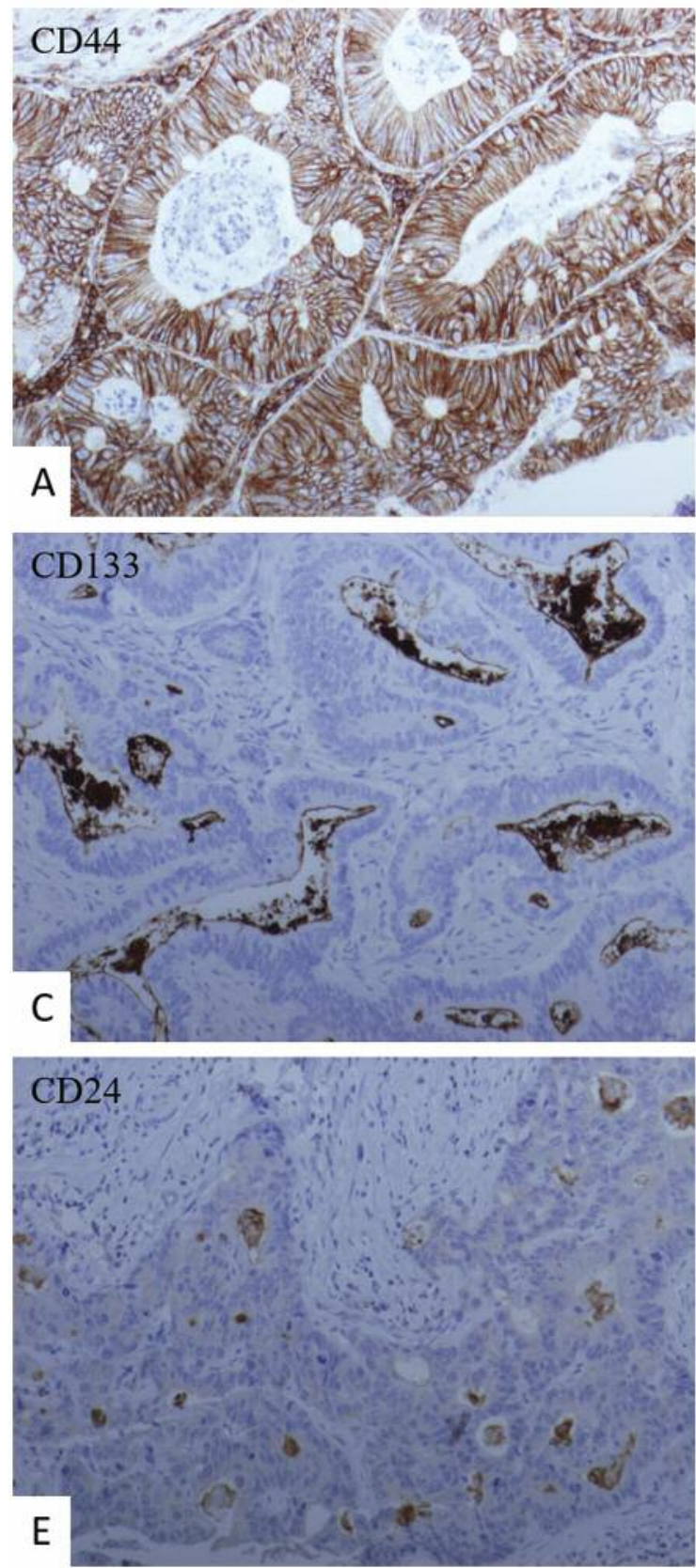

\section{Liver metastasis}
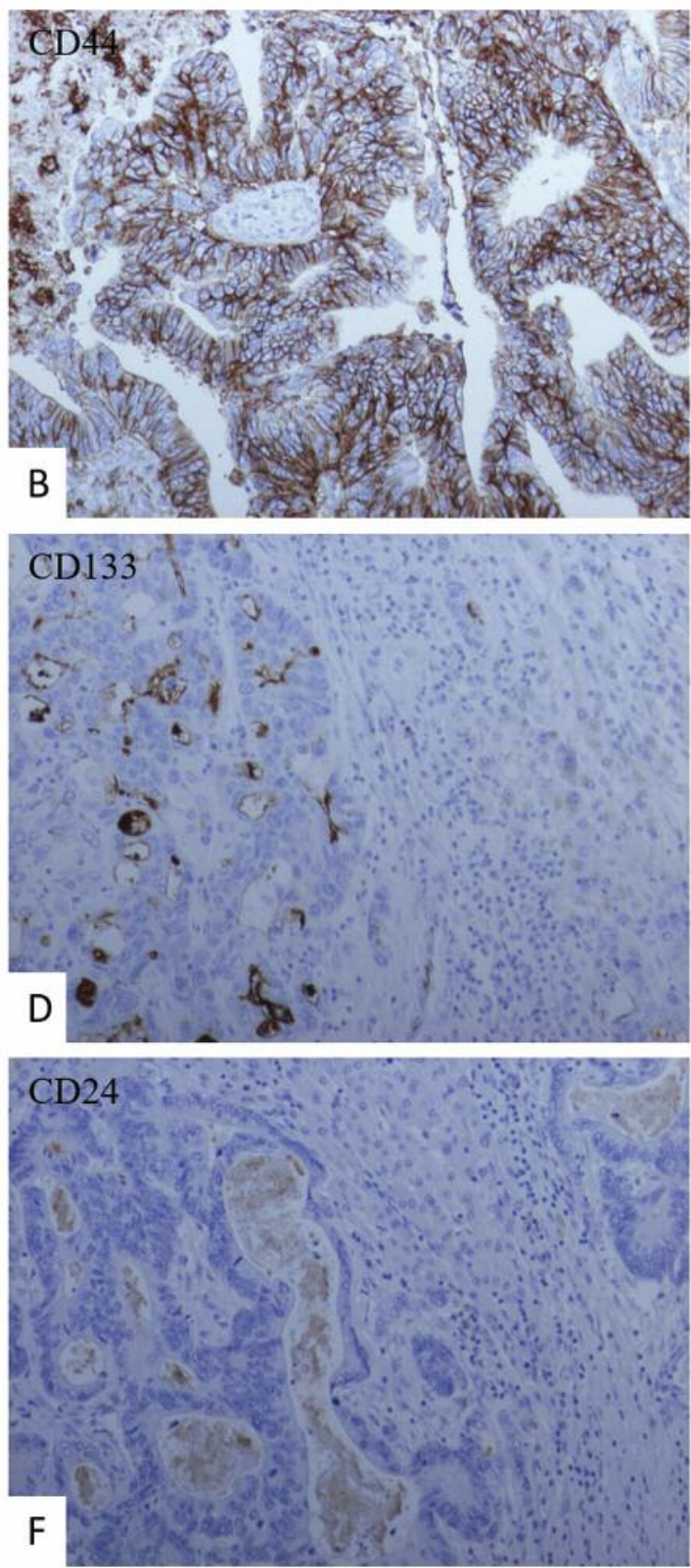

Figure 1. Microscopic images of primary colon cancer specimens staining positively for cancer stem cell markers CD44 (A), CD133 (C) and CD24 $(E)$, as well as specimens of liver metastases staining positively for cancer stem cell markers CD44 (B) CD133 (D) and CD24 (F).

statistically significant, whereas this difference was borderline significant for DFS $(p=0.051)$.

Cox proportional hazards regression was used to develop a multivariable survival model. Variables taken into consideration were age, gender, ASA-score, primary tumour specifics, synchronous versus metachronous liver metastases, number of liver metastases, diameter of the largest liver metastasis, preoperative CEA-level $>5 \mu \mathrm{g} / \mathrm{L}$, treatment with preoperative chemotherapy, extent and radicality of liver resection and severe complications (Clavien-Dindo classification (19) grade III or higher) after liver resection. Vascular invasion, perineural invasion and tumour deposits of the primary colon cancer, 

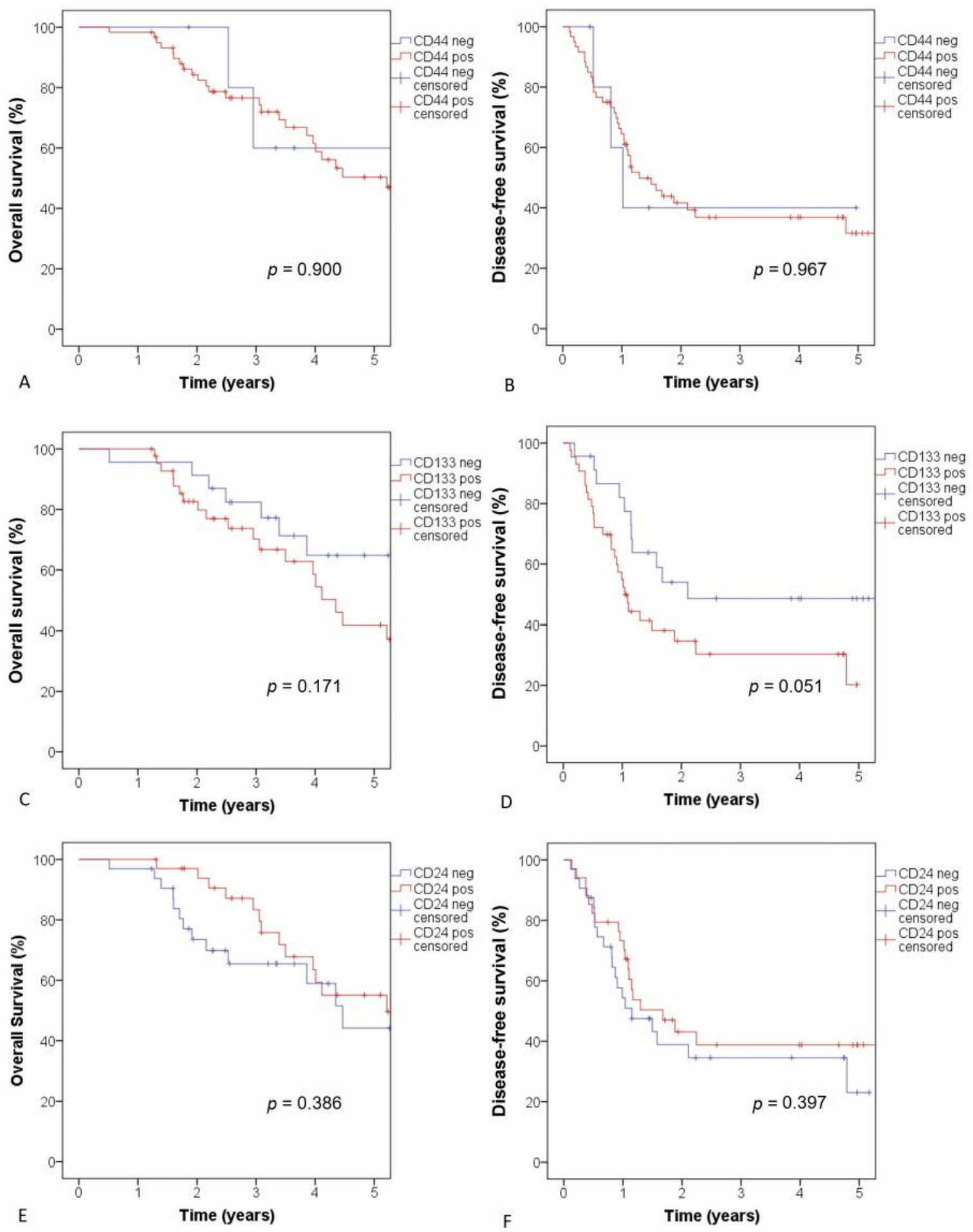

Figure 2. Kaplan-Meier curves of overall survival and disease-free survival after resection of colon cancer liver metastases, comparing patients with marker-positive and marker-negative liver metastases for cancer stem cell markers CD44 (A-B), CD133 (C-D) and CD24 (E-F), respectively.

synchronous liver metastases, major liver resection, preoperative chemotherapy, number of liver metastases and the occurrence of severe complications after liver resection were selected for both OS and DFS through univariate analysis. Additionally, age, ASA-score, T-stage and tumour grade of the primary cancer, as well as CEA-level $>5 \mu \mathrm{g} / \mathrm{L}$ were selected for $\mathrm{OS}$, and $\mathrm{N}$-stage 
and LNR of the primary cancer, as well as radicality of liver resection were selected for DFS. The mentioned variables and the expression state in the liver metastases for each CSC marker were included in the multivariate analysis. Through stepwise Cox proportional hazards regression, statistically significant prognostic variables were selected for both OS and DFS.

For OS, selected variables included perineural invasion and the presence of tumour deposits of the primary colon cancer. For DFS, identified variables were perineural invasion of the primary colon cancer, preoperative chemotherapy and CD133 expression in the liver metastasis. The individual selected variables and their hazard ratio (HR) are presented in Table III.

\section{Discussion}

Only a limited number of studies have previously analysed the expression of CSC markers in colonic liver metastases and how this predicts long-term outcome $(16-18,27)$. These reports mainly considered CD133 and CD44, whilst the current study, to our knowledge, is the first to analyse all three markers, including CD24, in colonic liver metastases and to assess their prognostic significance for outcome after hepatectomy.

More knowledge exists on these markers' expression in primary colon cancer and outcome after colon cancer treatment, with CD133 being claimed to be associated with both a worsened OS and DFS after colon cancer treatment (15). CD44 expression is found to predict a worsened OS (13), as is CD24 (14). However, another group found no relation between the expression of any of these markers and survival after colon cancer treatment, whilst they did conclude CD133 and CD24 to be correlated with invasiveness and differentiation (28).

Testing each marker for its influence on long-term outcome, we found CD133 expression to be most distinguishing, but only for DFS and with borderline significance. To correct for possible confounding factors, multivariate analysis was performed, and through this, CD133 was found to be an independent predictor for DFS. This is in accordance with the results found by Narita et al., who compared patients with early recurrence after liver resection with those with late recurrence, and found increased CD133 expression within the first group (27). In the present study, no relation between CD133 expression and OS was found, corresponding to the findings by Pitule et al. However, Pitule et al. neither found CD133 expression in liver metastases to be related to DFS (17), which contradicts the current results. Other contradicting results were reported by Yamamoto et al. who found CD133 expression in liver metastases to be associated with a better OS after hepatectomy (16) and Kishikawa et al. who reported both a better OS and DFS (18). Even though discrepancies exist between the results of the few available studies, the current findings are in accordance with results of the considerably larger available material on the prognostic value of CD133 expression in primary CRC,
Table III. Results of stepwise Cox proportional hazards regression showing the selected variables predicting overall survival and diseasefree survival after liver resection for colon cancer metastases.

\begin{tabular}{lccc}
\hline & HR & 95\%CI for HR & $p$-Value \\
\hline $\begin{array}{l}\text { Overall survival } \\
\quad \text { Perineural invasion }\end{array}$ & 3.915 & $1.447-10.588$ & 0.007 \\
$\quad \begin{array}{l}\text { Tumour deposits } \\
\text { Disease-free survival }\end{array}$ & 3.364 & $1.284-8.816$ & 0.014 \\
$\quad$ Perineural invasion & 3.058 & $1.441-6.493$ & 0.004 \\
$\quad$ Preoperative chemotherapy & 2.996 & $1.413-6.350$ & 0.004 \\
CD133 expression & 1.541 & $1.017-2.336$ & 0.041 \\
\hline
\end{tabular}

HR: Hazard ratio; CI: confidence interval.

where a meta-analysis associated this with a worse prognosis as well (15). Since there was no significant discordance between CD-133 expression in the primary colon cancer and the liver metastases in the present material, these findings are most likely associated.

In the present study, CD44 expression did not predict OS or DFS, a finding supported by two other reports on CSC markers in liver metastases $(16,17)$. Neither did we find CD24 expression to be of significance, but this has not been tested in the same manner previously.

For this study, a proportion of stained cells over $5 \%$ in IHC analysis was considered positive, in line with previous cut-off values for CSC markers in colorectal cancer (25) and one study on CD133 expression in CRC liver metastases (18). The latter also reported $56.8 \%$ of liver metastases to be CD133positive, comparable to the $64.2 \%$ in the current study. However, some authors used for example $10 \%$ (16) as a cutoff value. This is partly reflected in the different rates of CSC marker expression. The current study identified $91.0 \%$ of liver metastases as CD44-positive, whereas this value was $58.7 \%$ in a similar study using $10 \%$ as a cut-off value (16). The same researchers deemed $44.6 \%$ of liver metastases CD133positive, whereas $64.2 \%$ of liver tumours were considered CD133-positive in the present study. The varying cut-off values used in these studies on CSC marker expression in colonic liver metastasis contribute to differences in the results.

In this study, CD133 predicted DFS, but not OS. Even though DFS and OS are partly related, there are considerable differences. In DFS, any type of recurrence or spread is counted as an event, including small local recurrences for which the patient may be treated curatively. It is not uncommon for liver recurrences to be treated with a new curative resection, after which patients have been shown to have similar survival rates as after a first liver resection (29).

There are some limitations to this study, mainly the relatively small study population. However, since it is one of the first studies on this subject and probably the first to 
consider CD24 expression for outcome after liver resections, these results could prompt further investigation and steer larger, future studies into evaluating CD133 specifically and possibly additional novel markers.

In our study, a subpopulation of almost half the study group was also tested for marker expression in the primary tumour. Only few discordances for CD44 and CD133 were found, without statistical significance, whereas CD24 expression was significantly discordant. These discrepancies may be caused by a gradual change of the cancer cell population over time, thus causing different expression patterns in the metastatic tumour as compared to the primary colon cancer. Another hypothesis is that CSC marker expression could be influenced by chemotherapy given between the two operations, possibly through altering the expression per se, or due to the elimination of certain cells, changing the compilation of the cell population. In the last scenario one would expect higher rates of CSC expression in liver metastases occurring after chemotherapy treatment, since some studies have suggested that CSCs represent a source of tumour recurrence and resistance to chemotherapy (5). The sample size in this study was too small, though, to perform further analysis of the possible influence of chemotherapy treatment on the expression patterns in the metastatic tumour as compared to the primary colon cancer. However, since there were some differences in the individual CD133 expression in the primary cancer and the corresponding liver metastases, the results of this study can only be applied to the expression in liver metastases and therefore the marker's prognostic value will predominantly be of relevance when choosing adjuvant chemotherapy.

The present study shows CD133 expression in colonic liver metastases to be a predictive factor for worsened DFS after hepatectomy. If further studies can confirm our findings, CD133 could be used as a biomarker for risk stratification to select patients in need of more aggressive adjuvant therapy, but possibly also for the development of novel therapies. Since targeted therapy directed specifically against CSCs could possibly decrease the metastatic potential and chemotherapy resistance of cancer (30), CD133 may be a future target for novel treatment regimens for colonic liver metastases.

\section{Conflicts of Interest}

There are no conflicts of interest to be declared. No financial support was requested or received in the production of this article.

\section{References}

1 Statistics of Sweden. Cancer Incidence in Sweden 2013. http://www.socialstyrelsen.se, accessed November 2015.

2 Leporrier J, Maurel J, Chiche L, Bara S, Segol P and Launoy G: A population-based study of the incidence, management and prognosis of hepatic metastases from colorectal cancer. Br J Surg 93: 465-474, 2006.
3 Pawlik TM, Scoggins CR, Zorzi D, Abdalla EK, Andres A, Eng C, Curley SA, Loyer EM, Muratore A, Mentha G, Capussotti L and Vauthey JN: Effect of surgical margin status on survival and site of recurrence after hepatic resection for colorectal metastases. Ann Surg 241: 715-722, 2005.

4 Spelt L, Andersson B, Nilsson J and Andersson R: Prognostic models for outcome following liver resection for colorectal cancer metastases: A systematic review. Eur J Surg Oncol 38: 16-24, 2012.

5 Reya T, Morrison SJ, Clarke MF and Weissman IL: Stem cells, cancer, and cancer stem cells. Nature 414: 105-111, 2001.

6 Dou $\mathrm{J}$ and $\mathrm{Gu} \mathrm{N}$ : Emerging strategies for the identification and targeting of cancer stem cells. Tumour Biol 31: 243-253, 2010.

7 Dalerba P, Dylla SJ, Park IK, Liu R, Wang X, Cho RW, Hoey T, Gurney A, Huang EH, Simeone DM, Shelton AA, Parmiani G, Castelli $\mathrm{C}$ and Clarke MF: Phenotypic characterization of human colorectal cancer stem cells. Proc Natl Acad Sci USA 104: 10158-10163, 2007.

8 O'Brien CA, Pollett A, Gallinger S and Dick JE: A human colon cancer cell capable of initiating tumour growth in immunodeficient mice. Nature 445: 106-110, 2007.

9 Vermeulen L, Todaro M, de Sousa Mello F, Sprick MR, Kemper K, Perez Alea M, Richel DJ, Stassi G and Medema JP: Singlecell cloning of colon cancer stem cells reveals a multi-lineage differentiation capacity. Proc Natl Acad Sci USA 105: 1342713432, 2008.

10 Marhaba R and Zoller M: CD44 in cancer progression: adhesion, migration and growth regulation. J Mol Histol 35: 211-231, 2004.

11 Corbeil D, Roper K, Hellwig A, Tavian M, Miraglia S, Watt SM, Simmons PJ, Peault B, Buck DW and Huttner WB: The human AC133 hematopoietic stem cell antigen is also expressed in epithelial cells and targeted to plasma membrane protrusions. $\mathrm{J}$ Biol Chem 275: 5512-5520, 2000.

12 Jaggupilli A and Elkord E: Significance of CD44 and CD24 as cancer stem cell markers: an enduring ambiguity. Clin Dev Immunol 2012: 708036, 2012.

13 Huh JW, Kim HR, Kim YJ, Lee JH, Park YS, Cho SH and Joo JK: Expression of standard CD44 in human colorectal carcinoma: association with prognosis. Pathol Int 59: 241-246, 2009.

14 Weichert W, Denkert C, Burkhardt M, Gansukh T, Bellach J, Altevogt P, Dietel M and Kristiansen G: Cytoplasmic CD24 expression in colorectal cancer independently correlates with shortened patient survival. Clin Cancer Res 11: 6574-6581, 2005.

15 Chen S, Song X, Chen Z, Li X, Li M, Liu H and Li J: CD133 expression and the prognosis of colorectal cancer: a systematic review and meta-analysis. PLoS One 8: e56380, 2013.

16 Yamamoto S, Tanaka K, Takeda K, Akiyama H, Ichikawa Y, Nagashima $Y$ and Endo I: Patients with CD133-negative colorectal liver metastasis have a poor prognosis after hepatectomy. Ann Surg Oncol 21: 1853-1861, 2014.

17 Pitule P, Cedikova M, Daum O, Vojtisek J, Vycital O, Hosek P, Treska V, Hes O, Kralickova $M$ and Liska V: Immunohistochemical detection of cancer stem cell related markers CD44 and CD133 in metastatic colorectal cancer patients. BioMed Res Int 2014: 432139, 2014.

18 Kishikawa J, Kazama S, Oba K, Hasegawa K, Anzai H, Harada Y, Abe H, Matsusaka K, Hongo K, Oba M, Yasuda K, Otani K, Nishikawa T, Tanaka T, Tanaka J, Kiyomatsu T, Hata K, Kawai 
K, Nozawa H, Yamaguchi H, Ishihara S, Sunami E, Ushiku T, Kitayama J, Fukayama M, Kokudo N and Watanabe T: CD133 Expression at the Metastatic Site Predicts Patients' Outcome in Colorectal Cancer with Synchronous Liver Metastasis. Ann Surg Oncol 23: 1916-1923, 2016.

19 Dindo D, Demartines $\mathrm{N}$ and Clavien PA: Classification of surgical complications: a new proposal with evaluation in a cohort of 6336 patients and results of a survey. Ann Surg 240: 205-213, 2004.

20 Couinaud C: Le Foie. Etudes anatomiques et chirurgicales. Paris, Masson \& Cie, 1957.

21 Reddy SK, Barbas AS, Turley RS, Steel JL, Tsung A, Marsh JW, Geller DA and Clary BM: A standard definition of major hepatectomy: resection of four or more liver segments. HPB: the official journal of the International Hepato Pancreato Biliary Association 13: 494-502, 2011.

22 Compton C, Fenoglio-Preiser CM, Pettigrew N and Fielding LP: American Joint Committee on Cancer Prognostic Factors Consensus Conference: Colorectal Working Group. Cancer 88: 1739-1757, 2000.

23 Sobin LH, Gospodarowicz MK and Wittekind C: TNM Classification of Malignant Tumours (7th edition). Oxford, UK Blackwell Publishing Ltd, 2010.

24 Spelt L, Sasor A, Ansari D and Andersson R: Pattern of tumour growth of the primary colon cancer predicts long-term outcome after resection of liver metastases. Scand J Gastroenterol 51: 1233-1238, 2016.

25 Lugli A, Iezzi G, Hostettler I, Muraro MG, Mele V, Tornillo L, Carafa V, Spagnoli G, Terracciano L and Zlobec I: Prognostic impact of the expression of putative cancer stem cell markers CD133, CD166, CD44s, EpCAM, and ALDH1 in colorectal cancer. Br J Cancer 103: 382-390, 2010.
26 McShane LM, Altman DG, Sauerbrei W, Taube SE, Gion M, Clark GM and Statistics Subcommittee of the NCIEWGoCD: REporting recommendations for tumour MARKer prognostic studies (REMARK). Br J Cancer 93: 387-391, 2005.

27 Narita M, Oussoultzoglou E, Chenard MP, Fuchshuber P, Yamamoto T, Addeo P, Jaeck D and Bachellier P: Predicting early intrahepatic recurrence after curative resection of colorectal liver metastases with molecular markers. World J Surg 39: 11671176, 2015.

28 Choi D, Lee HW, Hur KY, Kim JJ, Park GS, Jang SH, Song YS, Jang KS and Paik SS: Cancer stem cell markers CD133 and CD24 correlate with invasiveness and differentiation in colorectal adenocarcinoma. World J Gastroenterol 15: 22582264, 2009.

29 Jonsson K, Grondahl G, Salo M, Tingstedt B and Andersson R: Repeated Liver Resection for Colorectal Liver Metastases: A comparison with primary liver resections concerning perioperative and long-term outcome. Gastroenterol Res Pract 2012: 568214, 2012.

30 Frank NY, Schatton T and Frank MH: The therapeutic promise of the cancer stem cell concept. J Clin Invest 120: 41-50, 2010.

Received September 28, 2017

Revised October 12, 2017

Accepted October 13, 2017 